\title{
REVIEW ARTICLE B cells and the microbiota: a missing connection in food allergy
}

\author{
Evelyn Campbell ${ }^{1,2}$, Lauren A. Hesser ${ }^{3}$ and Cathryn R. Nagler $\mathbb{D}^{2,3}$
}

Food allergies are a major public health concern due to their widespread and rising prevalence. The increase in food allergy is partially due to Western lifestyle habits which deplete protective commensal microbiota. These microbial perturbations can result in adverse host-microbe interactions, altering the phenotype of various immune cells and instigating allergic sensitization. Although B cells are critical to allergic pathology, microbial influences on B cells have been somewhat overlooked. Here, we focus on direct and indirect interactions between bacteria and $B$ cells and how such interactions regulate B-cell phenotype, namely antibody production ( $\lg \mathrm{A}, \lg \mathrm{E}, \lg \mathrm{G} 1$, and $\lg \mathrm{G} 4)$ and regulatory B-cell (Breg) function. Understanding how microbes modulate B-cell activity in the context of food allergies is critical to both tracing the development of disease and assessing future treatment options.

Mucosal Immunology (2021) 14:4-13; https://doi.org/10.1038/s41385-020-00350-x

\section{INTRODUCTION}

Food allergies are becoming an increasingly common problem across the globe, ${ }^{1}$ particularly in industrialized countries, including the United States. Over 32 million Americans are currently affected by food allergies, 2,3 including $~ 8 \%$ of US children. ${ }^{2}$ This rapid increase in prevalence within a single generation has led to the examination of environmental impacts on food allergy risk. Many twenty-first century lifestyle practices directly affect the microbiome, defined as the compilation of microorganisms including bacteria, fungi, and others that colonize the body's external surfaces. There is evidence that environmental factors which alter the composition of the microbiome in early life, including antibiotic use, Cesarean birth, and high-fat low-fiber diet, are responsible, in part, for the rise in food allergies. ${ }^{4,5}$ The human immune system is in constant communication with the microbiota on the skin, in the gut, and on other mucosal surfaces. It stands to reason that detrimental alterations to the microbiota, collectively termed dysbiosis, could be related to aberrant immune responses to food antigens. However, the specific mechanisms linking dysbiosis and food allergies are still unclear.

Food allergies as discussed in this review are characterized by type 2 immune responses to a food antigen and the production of immunoglobulin E (IgE) antibodies, although it should be noted that not all allergies are IgE-mediated. ${ }^{6}$ There are eight foods responsible for the majority of food allergies: eggs, milk, peanuts, tree nuts, soy, wheat, fish, and shellfish. ${ }^{6}$ Allergies to these foods are quite variable in average age of onset, resolution, and severity. ${ }^{7}$ For example, milk allergies often begin during infancy, but most will resolve during childhood. In contrast, peanut allergies often do not begin until childhood, but are known for their severity and commonly persist throughout the lifetime.

Sensitization to food allergens often occurs first upon ingestion, but there is growing evidence for the influence of disrupted skin barriers on allergic sensitization. ${ }^{8}$ In the gut, food allergens interact with or cross the intestinal epithelium, stimulating production of alarmins including IL-33 and IL-25. ${ }^{9}$ These alarmins initiate a type 2 microenvironment, mediated by type 2 innate lymphoid cells (ILC2s), which produce cytokines such as IL-13, IL-4, and IL-5. Local dendritic cells (DCs) residing mainly in Peyer's patches are primed by these cytokines, while picking up and processing food antigens. DCs present these antigens to naive lymphocytes either in the Peyer's patches or after migrating to mesenteric lymph nodes (MLN). Antigen-specific $\mathrm{CD}^{+}{ }^{+} \mathrm{T}$ cells activate GATA-3 to become type 2 helper cells (Th2) and further expand production of type 2 cytokines. Similarly, in these conditions B cells are licensed to undergo classswitch recombination (CSR) to $\operatorname{lgE}^{10}$ In addition to $\operatorname{lgE}$, other antibody classes such as IgA and IgG are now gaining recognition for their potential involvement in food allergies.

This review will primarily discuss microbial regulation of antibody production and B-cell function in the context of food allergy. When we consider the relationship between the humoral response and the microbiota, $\lg A$ is often the first thing to come to mind. $\lg A$ is able to bind to commensal bacteria and modulate the abundance and diversity of gut microbiota, ${ }^{11-14}$ just as the gut microbiota modulates the abundance and specificity of $\lg A{ }^{15}$ Through this reciprocal relationship, IgA contributes to homeostasis in a multitude of ways, and the absence of $\lg A$ in the gut may be as relevant to food allergies as the presence of $\operatorname{lgE} .^{16} \mathrm{In}$ fact, germ free (GF) mice, devoid of any microbiota, exhibit low levels of $\lg \mathrm{A}$ and, interestingly, high levels of $\lg \mathrm{E} .{ }^{17}$ This raises the question of whether and how bacteria regulate CSR to these two opposing immunoglobulins, and how this could relate to allergic responses. Without adequate signals from commensal microbiota, do mature $\lg \mathrm{A}$ - or $\mathrm{lgG}$-producing plasma cells ( $\mathrm{PCs}$ ) undergo further CSR to begin producing IgE? While this review will mainly discuss antibody-producing PCs, many other B-cell types may be relevant to the induction and long-term maintenance of food allergies including long-lived PCs or memory B cells.

Another subset of $B$ cells which may be relevant to the prevention of or recovery from food allergies is regulatory $B$ cells

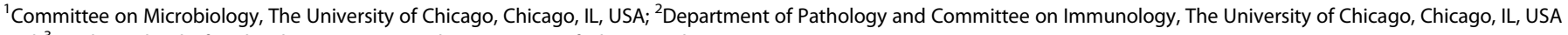
and ${ }^{3}$ Pritzker School of Molecular Engineering, The University of Chicago, Chicago, IL, USA

Correspondence: Cathryn R. Nagler (cnagler@bsd.uchicago.edu)

These authors contributed equally: Evelyn Campbell, Lauren A. Hesser
}

Received: 13 July 2020 Revised: 15 September 2020 Accepted: 2 October 2020

Published online: 26 October 2020 
Food allergies

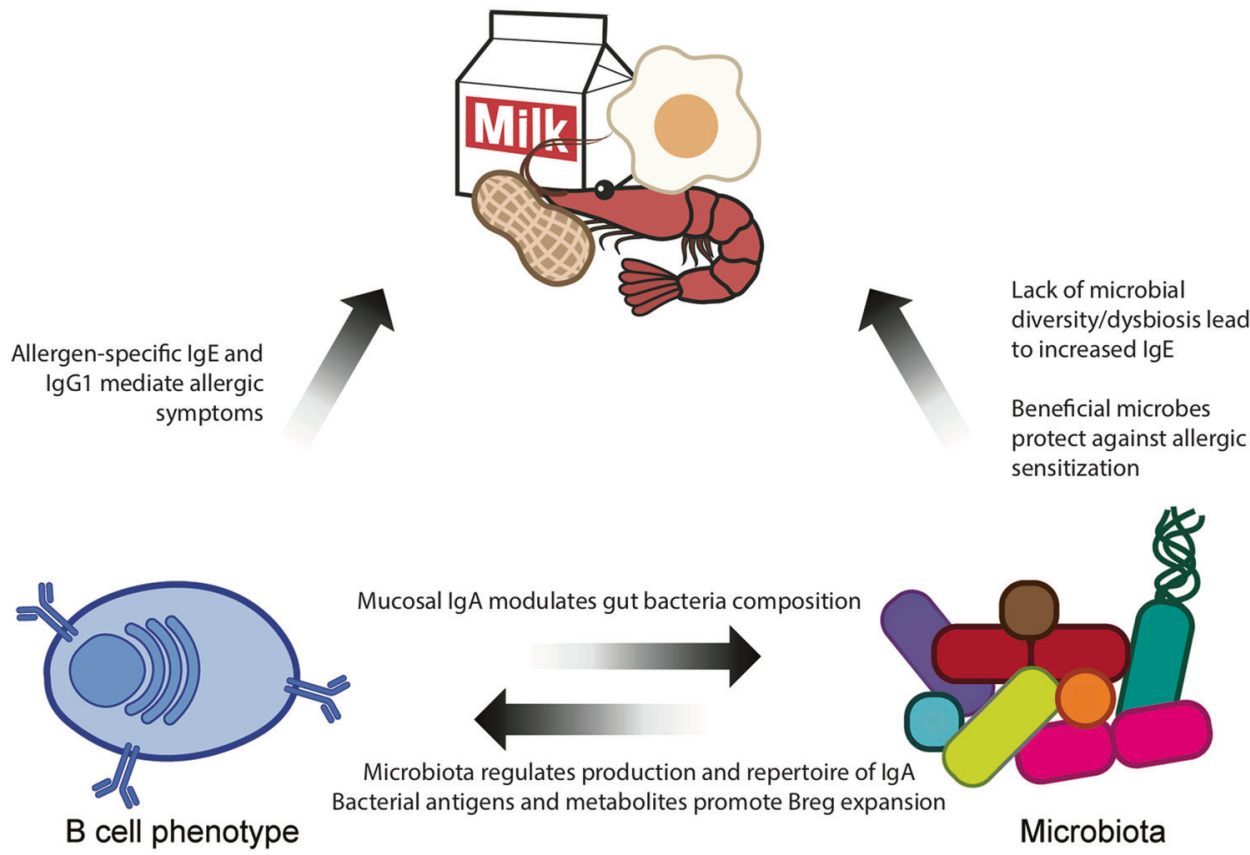

Fig. 1 The trilogy of food allergies. Individually, both the microbiota and B cells are known to play a role in allergic inflammation to dietary antigen. However, there has been a lack of appreciation of how the microbiota influences B-cell phenotype and vice versa. Specifically, bacterial antigens and metabolites stimulate $\lg \mathrm{A}$ production, regulate $\lg \mathrm{E}$ production, and induce expansion of Bregs. Conversely, IgA production and type 2 inflammatory responses can also affect bacterial community dynamics. In this review, we discuss how this relationship may contribute to food allergies.

(Bregs). Bregs, like their more commonly known regulatory T-cell (Treg) counterparts, produce IL-10 and have other immunosuppressive functions. Bregs are beginning to gain recognition for their involvement in food and airway allergies, ${ }^{18-20}$ and gut-local Bregs may be induced by the commensal microbiota to effectively prevent local inflammatory pathology. ${ }^{21}$

Overall, it is well known that B cells, and particularly lgE derived from these cells, are critical in regulating the allergic response to food. Separately, commensal bacteria have been identified for their roles in protecting against the development of food allergies. In this review we will discuss the evidence for microbial modulation of B-cell phenotype in the context of food allergy, particularly Ig class switching and induction of regulatory $B$ cells (Fig. 1).

Protective responses of $\lg \mathrm{A}$

IgA is the predominant immunoglobulin at mucosal surfaces, including the gastrointestinal tract, ${ }^{5}$ and the protective role of $\lg A$ in multiple disease pathologies has been demonstrated in numerous reports. ${ }^{22-24}$ Individuals with IgA deficiency, a common genetic immunological disorder that affects 1 in 700 Caucasians, have an altered gut microbiota compared to healthy controls, as well as increased susceptibility to allergy, celiac disease, and autoimmune diseases. ${ }^{14}$ By coordinating host-microbial interactions, IgA plays crucial immunoregulatory roles at the mucosa through several means such as facilitating mutualistic relationships with the commensal microbiota. ${ }^{23}$ quelling virulence of pathogens, ${ }^{24}$ and promoting beneficial immune responses. ${ }^{22}$ Further understanding of the exact mechanisms by which $\lg \mathrm{A}$ promotes intestinal health must be acquired to tap into its possible therapeutic properties.

Host-microbial symbiosis mediated by $\lg A$. IgA and commensal bacteria share a reciprocal relationship that contributes to the overall immune status of the host. Robust induction of $\lg A$ is dependent on bacterial antigens, as demonstrated by the observation that GF mice produce negligible $\operatorname{lgA}$ systemically and have fewer $\lg \mathrm{A}^{+} \mathrm{PCs}$ in the small intestine compared to specific pathogen free (SPF) mice. ${ }^{13,25}$ In fact, different strains of the same bacterial species can significantly influence the production of IgA in vivo, ${ }^{26}$ demonstrating the intricate nuances of microbial regulation of IgA. Furthermore, IgA can modulate the composition of the microbiota, and reduced concentrations of intestinal IgA correlate with decreased bacterial community diversity. ${ }^{14,27}$ Ruminococcaceae species have been reported to be overrepresented in IgA-deficient patients, and some data suggests that these bacteria are also enriched in food-allergic human infants. ${ }^{27-29}$ While these studies seem promising in identifying specific taxa that are regulated by $\operatorname{lgA}$, a lack of consistency in sequencing methods and analyses makes it difficult to compare results across studies. Since decreased bacterial diversity and dysbiosis are associated with food allergy, asthma, and other atopic diseases, maintenance of the homeostatic host-microbiota relationship by IgA has significant immunological implications which require further investigation.

$\lg A$ produced in the small intestine lamina propria by PCs is oligomerized via interactions with joining-chain (J-chain) to form dimeric secretory $\lg \mathrm{A}^{30}$ Secretory $\lg \mathrm{A}$ binds to the polymeric immunoglobulin receptor ( $\mathrm{plgR}$ ) located on the basolateral side of the epithelium. The plgR-IgA complex is endocytosed into the epithelial cell where plgR is enzymatically cleaved, forming the remaining protein that is referred to as the secretory component, which stabilizes and protects secretory $\lg A$ from proteolytic degradation by bacterial enzymes in the lumen. ${ }^{31}$ Once secretory $\lg \mathrm{A}$ is discharged to the apical side of the epithelium, it can interact with bacterial epitopes, such as lipopolysaccharide (LPS), flagellin, and capsular polysaccharides by Fab-dependent and Fab-independent mechanisms. While Fab-dependent mechanisms rely on the antigen-binding variable region of $\operatorname{lgA}$, Fabindependent mechanisms mediate antigen binding through other 
parts of $\lg \mathrm{A}$, including glycosylated sites on secretory component. $^{32}$ Fab-dependent and Fab-independent binding by $\lg \mathrm{A}$ averts bacteria-induced inflammation by preventing epithelial translocation and damage through multiple mechanisms including steric hindrance, immune exclusion, disruption of ligand-receptor interactions between bacteria and host epithelial cells, and alteration of bacterial gene expression and metabolism. ${ }^{33}$ These mechanisms mediate controlled antigen sampling and immunological education in early life.

Neonatal acquisition of passive IgA from breast milk prevents translocation of aerobic bacteria from the gut lumen to the MLN, shapes the composition of the intestinal microbiota, and regulates epithelial gene expression, which can influence susceptibility to intestinal inflammation later in life. ${ }^{22}$ IgA may play a crucial role in the "weaning reaction," a key period of immunological development around weaning age, which is induced based on the neonatal microbiota, components in breast milk, and the transition to solid foods. ${ }^{34}$ Several vital immune responses occur during this time, including the maturation of peripherally induced Tregs, which has been shown to be required for protection from chemically induced colitic and allergic intestinal inflammation later in life. ${ }^{34}$ Like the maturation of Tregs, induction of $\operatorname{lgA}{ }^{+} \mathrm{PCs}$ during this time period could have long-lasting protective effects. Conversely, the absence of a normal weaning reaction may be a result of early life perturbation of the microbiota. Early microbial disturbances along with exposure to pathogens during this crucial neonatal timeframe could result in adverse immune responses, including sensitization against food antigens that could last through adulthood. This may explain why mice and human infants that have reduced IgA-coated bacteria are more susceptible to allergic sensitization prior to or just following weaning. ${ }^{16,35}$ Thus, the beneficial interactions between $\lg A$ and the microbiota can have important implications for immune education and health.

$\operatorname{IgA}$ induction by bacterial and dietary antigens. The microbiota can induce IgA production via T-cell-independent (TI) and T-celldependent (TD) mechanisms. ${ }^{11}$ These two pathways of $\lg A$ induction, $\mathrm{TI}$ and TD, result in the generation of antibodies that typically have low affinity and polyreactivity or have high affinity and specificity, respectively. ${ }^{15}$

$\mathrm{TI}$ induction of $\operatorname{lgA}$ is mediated by innate-like B-1b cells, ${ }^{11,15}$ which exhibit no memory and produce low-affinity, polyreactive $\lg \mathrm{A}$, allowing for broad neutralization of microbial epitopes. ${ }^{13}$ Interactions between the commensal bacteria and host cells drive this broad IgA production, which has various immunological implications. In Peyer's patches, which are inductive sites for IgA in the small intestine, retinoic acid production by DCs following microbial stimulation is likely one of the main drivers of CSR from $\operatorname{lgM}$ to $\operatorname{lgA} .^{36}$ Short-chain fatty acids (SCFAs), such as acetate and butyrate, fermented from dietary fibers by the gut microbiota, have been shown to contribute to the induction of tolerogenic DCs and intestinal $\lg A$ secretion through G-protein coupled receptors (GPCRs) and enhance tolerance to food antigens. ${ }^{37-39}$ However, a recent study has shown that SCFAs can act as histone de-acetylation inhibitors that impair $\lg A$ production and other antibody responses. ${ }^{40}$ Despite this, the current literature supports the notion that bacteria can initiate a generally protective polyclonal $\lg \mathrm{A}$ response that helps to maintain homeostasis and resolve enteropathology. More studies will be needed to define the immunological contexts in which $\lg A$ is beneficial for intestinal health.

While the microbiota is required for normal IgA production, not all bacteria are equally capable of inducing it. Members of the Clostridia class, many of which produce SCFAs, are potent inducers of intestinal $\lg \mathrm{A}$, can decrease access of luminal food antigens into systemic circulation, and protect from allergic sensitization to food allergens ${ }^{41}$ (Table 1). By contrast, GF mice that are monocolonized with Bacteroides uniformis, an abundant intestinal microbe that does not produce SCFAs, poorly induce intestinal $\lg A$ and are not protected against sensitization to food. ${ }^{41}$ Bacterial proteins stimulate the epithelial cell lining of the intestine to produce secreted factors such as A proliferation-inducing ligand (APRIL), which regulates IL-10 and $\operatorname{lgA}$ production from $\mathrm{PCs}{ }^{42-44}$ Lactobacillus rhamnosus (LGG) produces a protein called p40 that can increase the expression of April in the small intestine epithelium and induce IgA production in vivo. ${ }^{42,43}$ Consumption of formula to which LGG has been added as a probiotic has been associated with accelerated acquisition of tolerance in cow's milk allergic (CMA) infants and children. ${ }^{28,45,46}$

TD induction of IgA largely elicits a specific response to antigen by $B 2$ cells. T-follicular helper (Tfh) cells in Peyer's patches interact with $\operatorname{lgM}^{+}$naive $\mathrm{B} 2$ cells and promote class switching to produce IgA via IL-21, retinoic acid, and CD40/CD40L interaction, which are specifically initiated by communication with bacteria. ${ }^{47,48} \mathrm{Com}$ mensal bacteria, including pathobionts, elicit IgA with specificity for particular bacterial epitopes. ${ }^{49} \operatorname{lgA}$ against specific bacterial antigens may assist in bacterial clearance and prevent translocation across the epithelium, and IgA can mark colitogenic, proinflammatory bacteria that contribute to intestinal inflammation. ${ }^{50,51}$ This may have implications for intestinal inflammatory responses resulting from allergic sensitization toward dietary antigen. Recently, Zhang et al. showed that the induction of high affinity, peanut-specific intestinal $\lg A$ is dependent on sensitization with a microbial-derived adjuvant (i.e. cholera toxin) and help from $\mathrm{CD}^{+}{ }^{+} \mathrm{T}$ cells, which does not include Tfh cells or T-follicular regulatory cells. ${ }^{52}$ As deficiency of $\operatorname{lgA}$ is associated with increased prevalence of atopic disease, ${ }^{53,54}$ these findings suggest that intestinal IgA may protect against allergic inflammation.

Studies examining the quantities of dietary antigen-specific $\lg \mathrm{A}$ have mainly focused on monomeric serum IgA, but the potential of serum IgA for regulating responses in the gut lumen is not clear. Food-specific $\lg \mathrm{A}$ has been detected at elevated levels in the serum of infants that become allergic later in life, ${ }^{55}$ as well as in mice that have been sensitized to peanut plus a mucosal adjuvant. ${ }^{52}$ Yet, others have shown that egg white-specific IgA2 is lower in the serum of allergic children than healthy controls, and only children that recover from the allergy show increased egg white-specific IgA2 over time. ${ }^{56}$ Furthermore, consumption of an antigen-free diet results in the reduction of both serum $\lg A$ and the frequency of food antigen-specific Tregs in the small intestine that are important for driving oral tolerance. ${ }^{57}$ These discrepancies suggest that measurement of serum IgA may not be useful as an indication of sensitization. Moreover, secretory lgA influences a more local and immediate response to intestinal perturbations and inflammation which is likely to be more relevant to food allergy pathology. Although the mechanisms are not fully elucidated, it is clear that $\lg A$ interacts with both bacteria and food antigens within the lumen of the gut, and this is likely to be important for maintaining intestinal homeostasis that suppresses allergic inflammation (summarized in Fig. 2).

$\lg E$ Induction and contributions to allergy $\lg E$ as a regulator of allergy. IgE is the canonical mediator of allergy. Individuals that are sensitized to food antigens produce antigen-specific IgE antibodies, which bind FcERI receptors on mast cells, basophils, or eosinophils. ${ }^{58}$ Upon re-exposure to the allergen, cell-bound antibodies are cross-linked by the antigen, causing the effector cells to degranulate and release histamine, eicosanoids, and many other mediators of allergic symptoms. ${ }^{58}$ Classically, type 2 cytokines IL-13 and IL-4 prompt B-cell CSR to $\lg \mathrm{E}^{10}$ but tissue damage and many other factors in the local milieu may also heighten the effect of these cytokines.

Type 2 immune responses are well characterized for their protective and necessary roles in both helminth infection ${ }^{59,60}$ and wound healing, ${ }^{61}$ yet the same has not been shown for $\operatorname{lgE}$. It has been hypothesized that the near-eradication of helminth infections 


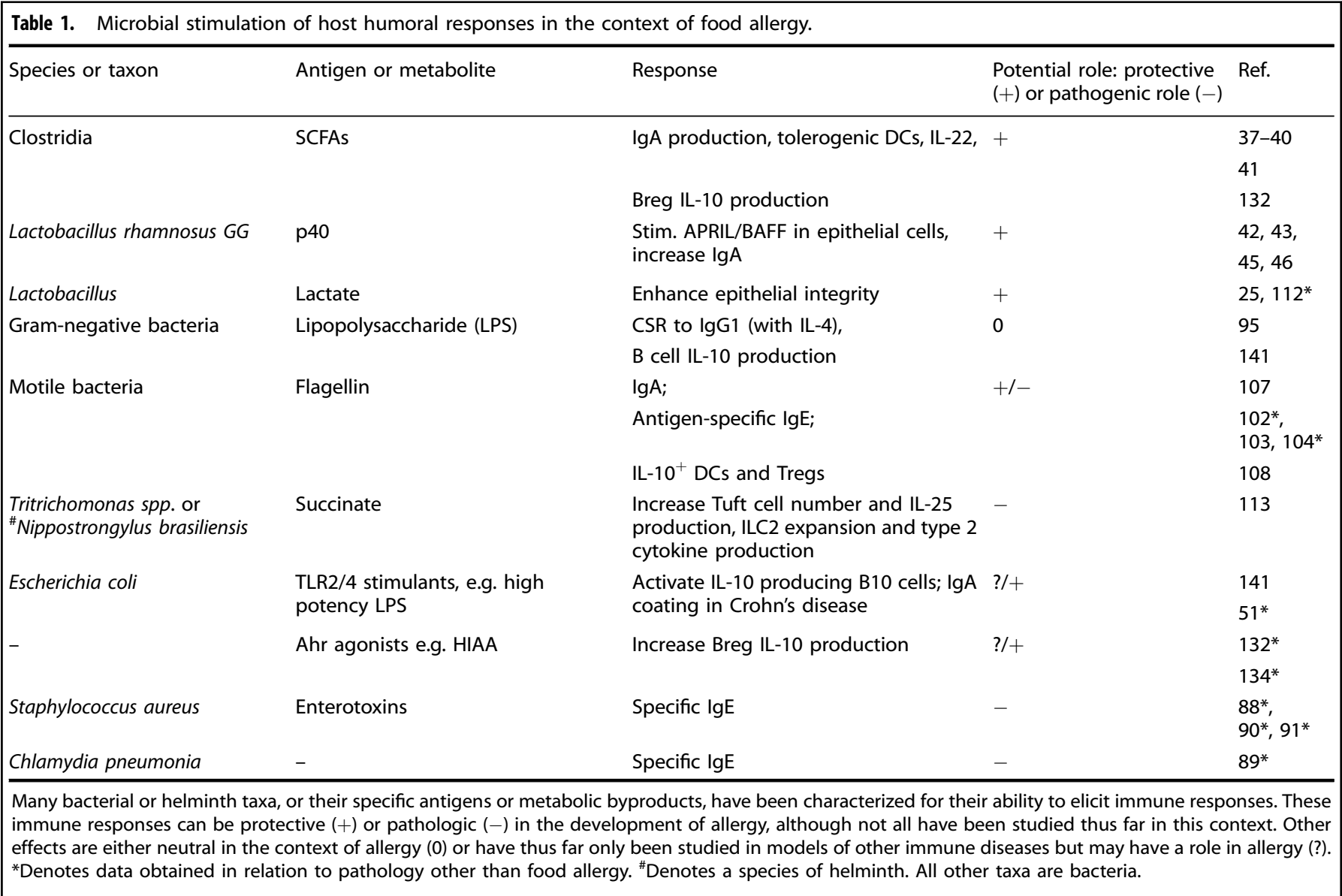

in modernized countries may be related to the rapid rise in the prevalence of allergy. ${ }^{62}$ Some studies have characterized beneficial functions of IgE in viral and parasitic infection or toxin exposure, but overall, there is minimal evidence for the "good-side" of IgE. ${ }^{63}$

Serum IgE concentrations are used as a clinical diagnostic test and are reported to have $\sim 95 \%$ sensitivity, but can have as little as $50 \%$ allergen specificity. ${ }^{64}$ In addition, serum IgE concentrations often do not correlate directly to the persistence or severity of an allergic response upon re-exposure to the allergen (i.e., a person (or mouse) with low allergen-specific IgE can have a severe allergic response, while another individual with high levels of allergenspecific $\lg E$ has no response). ${ }^{6,65}$ Recently, differences in $\operatorname{lgE}$ glycosylation patterns have been observed between healthy and peanut-allergic individuals. Shade et al. demonstrated that $\lg E$ from peanut-allergic individuals is decorated with sialic acid, which may be functionally relevant to the antibodies' capacity to potentiate an allergic response. $^{66}$

Quantifying IgE against a specific allergenic component, e.g. the peanut antigen Ara h 2, may be a better indicator of allergic severity than IgE against total peanut antigens. ${ }^{67}$ The same need for component specificity has been shown for cashew, hazelnut, wheat, and other allergens. ${ }^{67-69}$ Others demonstrate that quantifying the diversity of the antibody repertoire against cow's milk proteins is a good indicator of severity and persistence of milk allergy. ${ }^{70,71}$ Allergen component-specific IgE assays are clinically available, and analyses of antibody repertoire or glycosylation patterns may improve the accuracy of food allergy diagnostics.

Efforts are underway to prevent circulating lgE from inducing allergic responses. Omalizumab, a monoclonal antibody against $\lg \mathrm{E}_{1}$ is an FDA approved treatment for allergic asthma. ${ }^{72}$ Omalizumab is now also being tested in clinical trials in combination with oral immunotherapy to improve safety and efficacy in reducing the allergic response to food. ${ }^{73,74}$ Recent work has also shown that administration of soluble FceRl can inhibit circulating $\mathrm{lgE}$ and reduce the severity of IgE-mediated anaphylaxis in mice. ${ }^{75}$ While lgE-blocking treatments are exciting and can be quite effective, they do not address the root cause of aberrant $\lg \mathrm{E}$ production.

The factors that initiate IgE production are still unclear, as are the tissues in which this occurs. Classically, $\operatorname{lgE}^{+} \mathrm{PCs}$ reside mainly in the bone marrow or lymphoid tissues ${ }^{76}$ and circulate in low frequency in the blood of allergic individuals. ${ }^{77} \mathrm{New}$ evidence for local regulation suggests that some $\operatorname{lgE}$ may originate in the gut. ${ }^{78,79}$ Hoh et al. show that subjects with peanut allergy have many clones of peanut-specific antibodies (IgE and other isotypes) in biopsy samples from the stomach and duodenum, while healthy subjects do not. ${ }^{78}$ More distal regions of the gastrointestinal tract, like the ileum, were not analyzed in this study but would be interesting to examine in future studies. Analysis of clonal relationships between peanut-specific antibodies from the stomach and duodenum suggest that the majority of IgE clones are likely derived from antigen-experienced $B$ cells that have undergone local class switching from lgG or $\lg A$ to $\lg \mathrm{E}^{78,80}$ There are two pathways of CSR to lgE: either a direct switch to lgE or indirectly through $\lg A$ and $\operatorname{lgG} .^{81-83}$ The indirect method of CSR to lgE results in much higher affinity lgE than the direct method, ${ }^{83}$ as $\lg ^{+} \mathrm{B}$ cells are excluded from germinal centers and therefore do not undergo somatic hypermutation (SHM). ${ }^{84}$ It is likely that B cells which have undergone SHM with an IgG B-cell receptor (BCR) and then switched to IgE would produce antibodies with higher affinity. ${ }^{83}$ It will be interesting to learn how (and where) these pathways of CSR resulting in $\mathrm{IgE}$ are regulated in vivo.

In mice, $\lg E$ is the last gene in the immunoglobulin locus and it is not possible for $\operatorname{lgE}^{+} \mathrm{B}$ cells to undergo further class switching. 


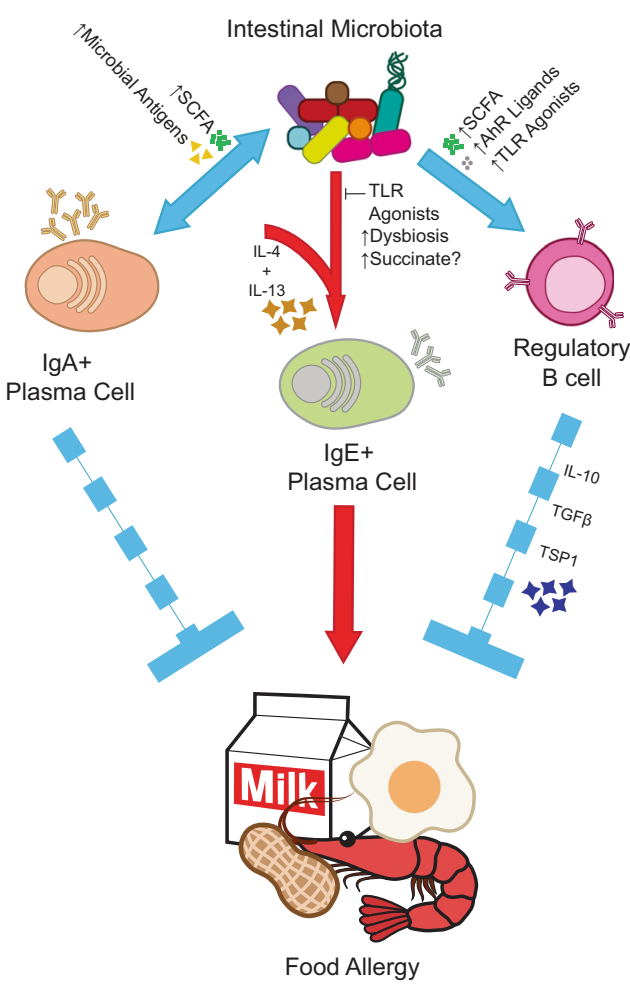

Fig. 2 Microbial antigens and products are able to influence B-cell phenotype. We focus on microbial regulation of three unique B-cell subsets: $\lg \mathrm{A}^{+}$plasma cells, $\operatorname{lgE}^{+}$plasma cells, and Bregs. Bacterialderived antigens and SCFAs directly interact with $B$ cells to induce $\lg A$ production through GPCR signaling and possibly other pathways or act indirectly through intestinal epithelial cells or DCs in T-cell-dependent and -independent pathways. TLR agonists, such as LPS and flagellin, can regulate $\lg \mathrm{E}$ production. The microbial metabolite succinate also promotes production of type 2 cytokines, which favors IgE CSR. SCFAs, AhR ligands, and TLR agonists may also stimulate immunosuppressive Bregs. Tolerogenic responses by $\lg \mathrm{A}^{+}$plasma cells and Bregs promote intestinal homeostasis and may help to dampen allergic inflammation (blue arrows), while secreted IgE mediates allergic symptoms and contributes to anaphylaxis (red terminator).

However, in humans, the gene for $\lg E$ is flanked by $\lg A 1$ and $\lg A 2$, which suggests that it may be possible to further class-switch $\lg \mathrm{E}^{+}$ $\mathrm{B}$ cells to a potentially tolerogenic $\lg \mathrm{A} 2$ isotype. ${ }^{78}$ Understanding the local regulation of CSR will be vital to manipulating and preventing aberrant lgE. Further studies are needed to examine the roles of $\operatorname{lgE}^{+}$memory $B$ cells and PCs, as well as their sites of induction and accumulation in specific mucosal tissues.

Direct regulation of IgE by the microbiota. Is there a role for bacteria in the regulation of IgE? Some evidence for this possibility comes from the observation that GF mice have elevated basal levels of systemic IgE even in the absence of sensitization. ${ }^{17}$ Feeding GF mice a specialized antigen-free diet is sufficient to reduce circulating lgE. ${ }^{85}$ These findings are in agreement with the hypothesis that dietary antigen-specific $\lg \mathrm{E}$ in food-allergic individuals is related to depleted or dysbiotic gut microbiota. In the absence of commensal bacteria, a corresponding decrease in bacteria-specific IgA may skew toward increased production of IgE. Antibacterial IgE has been examined, particularly in patients with asthma. The microbiota of patients with allergic asthma is often dysbiotic compared to healthy subjects, and this dysbiosis is partially characterized by the presence of atypical, potentially pathogenic bacteria. ${ }^{86,87} \mathrm{lgE}$ against many of these atypical bacteria has been characterized in the serum of individuals with allergic asthma (summarized in Table 1). ${ }^{88-91}$
Others have also studied whether controlled colonization with specific consortia of bacteria can reduce circulating lgE in GF mice. Wyss et al. demonstrated that GF mice colonized with complex consortia composed of a larger number of diverse species have reduced circulating lgE compared to those colonized with only a few species. ${ }^{92}$ However, their most complex controlled consortium, which contained 12 different species, could not fully reduce circulating $\mathrm{lgE}$ to concentrations comparable to SPF mice. ${ }^{92}$ Colonization with the feces of microbially replete SPF mice is sufficient to lower circulating lgE levels in GF mice, but this colonization must occur within a narrow window around weaning in order to have an effect. ${ }^{17}$ This provides further evidence for the role of microbiota during the weaning reaction ${ }^{34}$ and that this is a critical period of immune development.

We recently demonstrated a causal role for the healthy infant microbiota in protection against food allergy. We showed that GF mice colonized with feces from healthy infants were protected from the induction of allergen-specific $\lg G 1$ and $\lg \mathrm{E}$ as well as release of mast cell protease (mMCPT-1) following sensitization with the cow's milk allergen $\beta$-lactoglobulin (BLG). ${ }^{93}$ Conversely, mice colonized with the feces of CMA infants retained high levels of BLG-specific $\lg E$ and $\lg \mathrm{G} 1$ and exhibited potent allergic responses, similar to GF mice. ${ }^{93}$ We then identified a single bacterial species in the healthy infant microbiota that was sufficient to reduce the allergic response, including decreasing BLG-specific antibodies in monocolonized mice. ${ }^{93}$ These findings suggest that the production of both bulk and food antigenspecific IgE may be related to a lack of signals derived from commensal bacteria, which are present in healthy infants and SPF mice.

One way in which bacteria may interact directly with B cells is through stimulation of toll-like receptors (TLRs). Unlike most $T$ cells, B cells express a variety of TLRs that recognize bacterial molecules. TLR stimulation or BCR cross-linking by bacterial antigens can activate $B$ cells and induce immunoglobulin class switching in a T-cell-independent manner. ${ }^{94}$ LPS, a component of many Gram-negative bacterial cell membranes, binds TLR4 and can induce CSR to IgG1 in the presence of IL-4. ${ }^{95}$ We previously showed that TLR4-deficient mice develop a systemic allergic response after sensitization with peanut plus cholera toxin, including elevated peanut-specific $\operatorname{lgE}$, compared to TLR4sufficient mouse strains. ${ }^{96}$ This was the first evidence for bacterial regulation of an allergic response to food. However, it is thus far unknown exactly which cell type(s) are involved in TLR4 signaling in the context of food allergy. Healthy human B cells generally express low levels of TLR4, but expression is increased in patients with Crohn's disease. ${ }^{97}$ In addition, asthmatic patients with high circulating levels of $\operatorname{lgE}$ exhibit higher percentages of TLR4 ${ }^{+}$ macrophages compared to those with normal levels of circulating IgE. ${ }^{98}$ These patterns have not yet been examined in food-allergic patients, but stimulation with IL-4 in addition to anti-lg $\mu$ and antiCD40 significantly increases TLR4 mRNA and surface protein expression in human $B$ cells in vitro, ${ }^{97}$ providing some evidence for synergy between type 2 responses and TLR4 regulation on $B$ cells.

There is also some data suggesting that the physiological and immunological responses that are associated with food allergy could alter the composition of the gut microbiota. Gut pathology can physically disrupt the microbial community, as is the case in diarrhea (which can be a symptom of food allergy). Mild osmotic diarrhea in mice alters mucus structure, induces innate and adaptive immune responses, and diminishes several bacterial taxa. ${ }^{99}$ Type 2 immune signaling also seems to alter the microbial community (directly or indirectly), as is the case for IL4Ra ${ }^{F 709}$ mice, which have a gain-of-function mutation in the IL-4 receptor resulting in constitutively active IL-4 signaling. These mice have a unique, dysbiotic microbial signature as compared to wild-type mice. ${ }^{100}$ Sensitizing these mice with ovalbumin plus cholera toxin 
further alters the microbiota. ${ }^{100}$ However, these studies are limited due to lack of littermate controls, which are necessary to normalize founder microbial communities across experimental groups. Subsequent studies show that treatment of SPF mice with therapeutic bacteria, identified from healthy infants, effectively prevents the allergic response in these dysbiotic SPF IL4Ra ${ }^{F 709}$ mice as well as GF mice. ${ }^{35}$ While this is interesting evidence for the role of gut bacteria in regulating the allergic response, it remains unclear why the bacterial community, particularly in the IL4Ra ${ }^{F 709}$ mice, changes upon sensitization and which mechanisms are regulating the microbiota.

Innate mechanisms of microbiota-immune interactions in food allergy

The intestinal epithelium is in constant contact with the microbiota and acts as a barrier between the gut lumen and the lamina propria. Intestinal epithelial cells express many pattern recognition receptors including TLR5 which recognizes the protein flagellin, the major component of flagella on motile bacteria. ${ }^{101}$ There is evidence that flagellin could either exacerbate or suppress allergy and allergic asthma, ${ }^{102-104}$ possibly due to differences in the tissue or manner of exposure. Flagellin influences several immuno-protective responses in the gut including IL-22 production ${ }^{105,106}$ and long-term production of flagellin-specific IgA. ${ }^{107}$ Antigen-flagellin fusion protein constructs have been explored as mucosal vaccines to prevent food allergy, and have shown that TLR5 signaling in DCs elicits IL-10 production and reduction of antigen-specific IgE. ${ }^{108,109}$

Alterations to the cell-type composition or integrity of the intestinal epithelium likely also contribute to the onset of allergy. A bacteria-induced IL-22 response is critical to the maintenance of epithelial barrier function. ${ }^{4}$ GF and antibiotic-treated mice produce low levels of IL-22 in the ileum, and the detection of intact peanut protein in the serum after intragastric administration suggests that their barrier function has also been compromised. ${ }^{41}$ A large body of work has asked what makes some food proteins more allergenic than others, and it seems that resistance to degradation by digestive enzymes may be a key factor in allergenicity. ${ }^{110,111}$ Intact, undigested food antigens which penetrate the intestinal barrier could directly bind BCRs, initiating or perpetuating the allergic response. ${ }^{4,110}$

In addition, many cell types which make up the intestinal epithelium are responsive to bacterial metabolites. For example, lactate derived from Bifidobacterium or Lactobacillus species increases the number of intestinal stem cells, Paneth cells, and goblet cells in the ileum, which aid in protection from druginduced intestinal injury. ${ }^{112}$ Butyrate and acetate, bacterialderived SCFAs, signal through GPCRs (namely GPR43) on epithelial cells, resulting in protection from food allergy by a variety of pathways, as discussed. ${ }^{37}$ Conversely, succinate, a metabolite produced by bacteria and helminths, also affects the composition of the intestinal epithelium by expanding Tuft cells, which produce IL-25 and induce downstream ILC2 expansion and production of $\mathrm{IL}-13$ and $\mathrm{IL}-5 .{ }^{113}$ Overall, interaction between commensal bacteria and the intestinal epithelium is likely to have many downstream effects on the development of food allergy.

Opposing roles of IgG subclasses in allergy

IgG antibodies play unique roles in allergy. IgG1 is recognized for functional, "proinflammatory" roles, including in the context of allergy. In mice, IgG1 (like IgE) can elicit allergic anaphylaxis by binding effector cells and prompting release of proteases, including platelet activating factor. ${ }^{114-116} \operatorname{lgG} 1$ serves as an additional marker of the allergic response, and food antigenspecific $\operatorname{lgG} 1$ and lgE often show similar expression patterns in mouse models of allergy. ${ }^{41,52,93}$ lgG1 has been measured in the saliva of children with allergies ${ }^{117}$ and the serum of adults who experience anaphylaxis but do not exhibit increased $\lg \mathrm{E}_{1}^{115}$ demonstrating that $\operatorname{lgG} 1$ may be critical to non-lgE-mediated allergy. Though it may play a more minor role compared to $\lg \mathrm{E}$, $\operatorname{lgG} 1$ can contribute to inflammatory responses that are induced by allergens.

In contrast to $\lg \mathrm{G} 1$, another subclass, IgG4, may play protective roles in allergy in humans. ${ }^{118}$ Unlike other classes of IgG, lgG4 seems to be a unique "anti-inflammatory" antibody class that is produced mainly by Bregs. ${ }^{119}$ lgG4 was first measured in high abundance in the serum of beekeepers that became tolerant to bee venom, ${ }^{120}$ and now is recognized in food-allergic patients following oral immunotherapy, ${ }^{121,122}$ treatment with the anti-lgE antibody omalizumab, ${ }^{123}$ or both. ${ }^{124,125}$ IgG4's inability to fix complement contributes to its low inflammatory capacity. ${ }^{126,127}$ It also binds receptors FCYR1 (CD64) and the inhibitory receptor FCYRIlb, ${ }^{128,129}$ and in doing so can reduce the activity of mast cells. ${ }^{130}$ This weak receptor binding and cellular activation capacity is likely due in part to the fact that lgG4 is often monovalent. ${ }^{127}$ lgG4 can bind free allergen in the serum (competing with lgE), effectively flagging the antigen for clearance from the blood without eliciting a full cellular response. This antigen competition between lgG4 and $\operatorname{lgE}$ may be a critical mechanism of reducing the allergic response, particularly in the case of immunotherapy. While lgG4/lgE ratios may have some accuracy in predicting the allergic response to food, ${ }^{131}$ not all evidence agrees. ${ }^{132} \mathrm{~A}$ better understanding of the exact role(s) of IgG subclasses in food allergy, both protective and pathogenic, will give greater insight into complex interactions which occur during the allergic response.

Microbial influences on regulatory B cells in food allergy Induction of Tregs has been a topic of great interest in the treatment of food allergy. However, B cells, specifically Bregs, also have immunosuppressive functions in numerous inflammatory and disease models, including food allergies. ${ }^{18,19,133-135}$ Bregs are diverse in ontogeny and can arise from multiple B-cell subsets, including B1a cells, marginal zone B cells, and PCs. ${ }^{21,136}$ Because of this plasticity, consistent surface markers and transcription factors have not yet been identified to distinguish Bregs from other B cell subsets. Bregs produce several immunosuppressive cytokines including IL-10, TGF $\beta$, and TSP1 which assist in their function to repress T-cell mediated inflammation, enhance Treg function, induce tolerogenic DCs, and alter the phenotype of other local $B$ cells. ${ }^{19}$ In addition, surface markers such as FasL and PD-L1, which have immunosuppressive effects in T cells, are often upregulated in Bregs. ${ }^{137,138}$ This diversity in surface markers and cytokines produced by Bregs may be attributed to inflammatory signals specific to the local environment along with other stimuli, such as TLR agonists and metabolites derived from the microbiota.

Examination of Breg phenotypes in allergic airway and food allergy models showcases utilization of similar suppression mechanisms in these different atopic diseases. In allergic airway models, IL-10-secreting CD9 ${ }^{+} \mathrm{B}$ cells and $\mathrm{CD} 19^{+} \mathrm{B}$ cells are critical for dampening type 2 inflammation and inducing Tregs, respectively. ${ }^{18-20,134}$ Models for food allergy have shown that TSP1-secreting CD19 ${ }^{+}$and TGF $\beta$-secreting CD5 ${ }^{+} \mathrm{CD} 19^{+} \mathrm{CX} 3 \mathrm{CR} 1^{+}$ Bregs in the small intestine are critical for conferring protection from allergic sensitization to dietary antigen. ${ }^{18,20}$ TSP1 production from Bregs is required to induce tolerogenic TGF $\beta$-secreting DCs, which are known to suppress inflammatory responses in the gut. $^{139,140}$ In addition, Bregs can produce TGF $\beta$ themselves which acts directly on helper $T$ cells to suppress activation and increase Treg differentiation. ${ }^{19} \mathrm{IL}-10$ production by $\mathrm{CD}^{+}$ Bregs in the MLN suppresses allergic responses to cow's milk casein by inducing local Foxp3 ${ }^{+}$Tregs. $^{20}$ While there are various types of Bregs, these studies show many ways that tissue-resident Bregs can enact similar mechanisms to promote tolerogenic responses by secreting effector cytokines that act on other immune cells. 
Bacterial signals can also influence Breg phenotype, potentially through interaction with TLR agonists or SCFAs. GF mice have lower numbers of total B cells in the colon tissue compared to SPF mice, and colonization of GF mice with feces from SPF mice increases B-cell number and total IL-10 in the colon. ${ }^{141}$ TLR interaction with various commensal bacteria can differentially prime Bregs to produce IL-10. A recent study by Mishima et al. shows differing capacities of various bacterial lysates to induce IL10 in GF and SPF B cells from the MLN; lysates from E. coli elicited the highest IL-10 yield. ${ }^{141}$ Another study by Maerz et al. demonstrated that monocolonization with $E$. coli promotes increased IL-10 production by B10 cells and aids in protection from dextran sodium sulfate-induced intestinal damage, while monocolonization with Bacteroides vulgatis does not. ${ }^{142}$ Both of these studies claim that immunosuppression induced by Bregs is dependent on bacterial signaling through TLR2 and/or TLR4. However, these experiments were not littermate controlled, so founder bacteria in these mice may be significantly different across litters and groups, confounding results. Nonetheless, these claims align with previous work showing that Finnish and Estonian infants have increased susceptibility to allergy and autoimmune disease compared to their Russian counterparts, which corresponds with an increase of Bacteroides species in the first year of life. ${ }^{143}$ Structural variations in LPS from Bacteroides species compared to that of $E$. coli result in decreased immunogenicity of Bacteroides, which may prevent the induction of microbialinduced tolerogenic responses in Finnish and Estonian infants. ${ }^{143}$ The role of Bregs in this study was not investigated, but it is interesting to consider the contribution of TLR signaling in promoting tolerogenic functions of Bregs, especially during early life education of the immune system. Bacterial-derived AhR agonists and SCFAs, particularly butyrate, can also influence IL10 production in Bregs. Mechanistically, butyrate can increase the quantity of the serotonin-derived metabolite 5-hydroxyindole-3acetic acid (5-HIAA), which binds to the Aryl Hydrocarbon receptor (AhR). ${ }^{133}$ AhR acts a transcriptional regulator for Bregs, increasing production of IL-10 and suppression of proinflammatory cytokines TNFa and IL-6. ${ }^{135}$

Various microbial-derived cues from the local environment are important for shaping Breg phenotype. These stimuli, whether they originate from bacterial metabolic products or bacterial antigens, can be redundant in promoting Breg function (i.e., IL-10 production), but this redundancy allows many members of the microbiota to promote immunosuppression via Bregs. Thus, it is intuitive that lack of a microbiota or treatment with antibiotics would reduce Bregs and their suppressive capabilities. ${ }^{133}$ Further studies are needed to elucidate the role of bacterial metabolites in promoting oral tolerance through Bregs.

\section{CONCLUSION}

As demonstrated in numerous reports within the literature, there are strong links between B-cell phenotype, composition of the microbiota, and allergic sensitization to dietary antigens (Fig. 2). It is widely known that those who are suffering from genetic or drug-induced IgA deficiency have increased susceptibility to allergic diseases, including food allergies, and have an altered, intestinal microbial community. ${ }^{14,27}$ Microbial antigens or metabolic products, such as TLR agonists or SCFAs, can alter the production and repertoire of secreted immunoglobulins via epigenetic, transcriptomic, and metabolic pathway modulations. Examination of innate immune cell pathways and their influences on allergic inflammation and B-cell phenotype may be another possible avenue to consider. In allergic airway models, TLR ligands of bacterial origin have been shown to have differential effects on various adaptive and innate immune cells, including B cells, and play a role in IgE secretion and allergic inflammation. ${ }^{102,104}$ This is likely relevant in the context of dietary allergic sensitization, as we have already seen that signaling through innate pathways by Clostridia-derived ligands confers protection. ${ }^{41}$ One conundrum that remains is the opposing roles that $B$ cells play in food allergy pathology. B cells can both propagate sensitization to food allergens and dampen inflammatory responses, and studying the influences of the microbiota adds another layer of complexity. Do the direct and indirect effects of the microbiota on B cells contribute to food allergy? Or are changes in microbial community structure a byproduct of B-cell phenotype in an allergic context? The crux of these questions is whether dysbiosis is causative or correlative with allergic sensitization to dietary antigen. Future studies that address these questions will be pivotal to both understanding and eventually treating the food allergy epidemic.

\section{ACKNOWLEDGEMENTS}

We thank A. Kemter, S. Cao, A. Mirmira, R. Patry, and L. Maccio-Maretto for critical review and discussion of this manuscript. This work was supported by The Sunshine Charitable Foundation and NIH R01s Al106302 and Al146099.

\section{AUTHOR CONTRIBUTIONS}

E.C. and L.A.H. selected the topic(s) to be reviewed and drafted the manuscript. C.R.N. revised and edited the manuscript.

\section{ADDITIONAL INFORMATION}

Competing interests: C.R.N. is co-founder and president of ClostraBio, Inc. E.C. and L.A.H. declare no competing interests.

Publisher's note Springer Nature remains neutral with regard to jurisdictional claims in published maps and institutional affiliations.

\section{REFERENCES}

1. Warren, C. M., Jiang, J. \& Gupta, R. S. Epidemiology and burden of food allergy. Curr. Allergy Asthma Rep. 20, 6 (2020).

2. Gupta, R. S. et al. The public health impact of parent-reported childhood food allergies in the United States. Pediatrics 142, e20181235 (2018).

3. Gupta, R. S. et al. Prevalence and severity of food allergies among US adults. JAMA Netw. Open 2, e185630 (2019).

4. Wesemann, D. R. \& Nagler, C. R. The microbiome, timing, and barrier function in the context of allergic disease. Immunity 44, 728-738 (2016).

5. Iweala, O. I. \& Nagler, C. R. The microbiome and food allergy. Annu. Rev. Immunol. 37, 377-403 (2019).

6. Sicherer, S. H. \& Sampson, H. A. Food allergy: a review and update on epidemiology, pathogenesis, diagnosis, prevention, and management. J. Allergy Clin. Immunol. 141, 41-58 (2018).

7. Savage, J. \& Johns, C. B. Food allergy: epidemiology and natural history. Immunol. Allergy Clin. N. Am. 35, 45-59 (2015).

8. Sampson, H. A. et al. Mechanisms of food allergy. J. Allergy Clin. Immunol. 141, 11-19 (2018)

9. Peterson, L. W. \& Artis, D. Intestinal epithelial cells: regulators of barrier function and immune homeostasis. Nat. Rev. Immunol. 14, 141-153 (2014).

10. Tong, P. \& Wesemann, D. R. Molecular mechanisms of IgE class switch recombination. Curr. Top. Microbiol. Immunol. 388, 21-37 (2015).

11. Macpherson, A. J. et al. A primitive T cell-independent mechanism of intestinal mucosal IgA responses to commensal bacteria. Science 288, 2222-2226 (2000).

12. Moor, K. et al. High-avidity IgA protects the intestine by enchaining growing bacteria. Nature 544, 498-502 (2017).

13. Bunker, J. J. et al. Natural polyreactive $\lg \mathrm{A}$ antibodies coat the intestinal microbiota. Science 358, eaan6619 (2017).

14. Catanzaro, J. R. et al. IgA-deficient humans exhibit gut microbiota dysbiosis despite secretion of compensatory IgM. Sci. Rep. 9, 13574 (2019).

15. Bunker, J. J. et al. Innate and adaptive humoral responses coat distinct commensal bacteria with immunoglobulin A. Immunity 43, 541-553 (2015).

16. Dzidic, M. et al. Aberrant IgA responses to the gut microbiota during infancy precede asthma and allergy development. J. Allergy Clin. Immunol. 139, 1017-1025.e14 (2017).

17. Cahenzli, J., Köller, Y., Wyss, M., Geuking, M. B. \& McCoy, K. D. Intestinal microbial diversity during early-life colonization shapes long-term lgE levels. Cell Host Microbe 14, 559-570 (2013). 
18. Liu, Z. Q. et al. Tolerogenic CX3CR1+ B cells suppress food allergy-induced intestinal inflammation in mice. Allergy 68, 1241-1248 (2013).

19. Braza, F. et al. A regulatory CD9+ B-cell subset inhibits HDM-induced allergic airway inflammation. Allergy 70, 1421-1431 (2015).

20. Kim, A.-R. et al. Mesenteric IL-10-producing CD5+ regulatory B cells suppress cow's milk casein-induced allergic responses in mice. Sci. Rep. 6, 19685 (2016).

21. Rosser, E. C. \& Mauri, C. Regulatory B cells: origin, phenotype, and function. Immunity 42, 607-612 (2015).

22. Rogier, E. W. et al. Secretory antibodies in breast milk promote long-term intestinal homeostasis by regulating the gut microbiota and host gene expression. Proc. Natl Acad. Sci. USA 111, 3074-3079 (2014).

23. Macpherson, A. J., Yilmaz, B., Limenitakis, J. P. \& Ganal-Vonarburg, S. C. IgA function in relation to the intestinal microbiota. Annu. Rev. Immunol. 36, 359-381 (2018).

24. Proietti, M. et al. ATP released by intestinal bacteria limits the generation of protective IgA against enteropathogens. Nat. Commun. 10, 250 (2019).

25. Kozakova, $\mathrm{H}$. et al. Colonization of germ-free mice with a mixture of three lactobacillus strains enhances the integrity of gut mucosa and ameliorates allergic sensitization. Cell. Mol. Immunol. 13, 251-262 (2016).

26. Yang, C. et al. Fecal IgA levels are determined by strain-level differences in bacteroides ovatus and are modifiable by gut microbiota manipulation. Cell Host Microbe 27, 467-475.e6 (2020).

27. Fadlallah, J. et al. Microbial ecology perturbation in human IgA deficiency. Sci. Transl. Med. 10, eean1217 (2018).

28. Berni Canani, R. et al. Lactobacillus rhamnosus GG-supplemented formula expands butyrate-producing bacterial strains in food allergic infants. ISME J. 10, 742-750 (2016).

29. Fazlollahi, M. et al. Early-life gut microbiome and egg allergy. Allergy 73, 1515-1524 (2018)

30. Kumar, N., Arthur, C. P., Ciferri, C. \& Matsumoto, M. L. Structure of the secretory immunoglobulin A core. Science 367, 1008-1014 (2020).

31. Turula, H. \& Wobus, C. The role of the polymeric immunoglobulin receptor and secretory immunoglobulins during mucosal infection and immunity. Viruses $\mathbf{1 0}$, 237 (2018).

32. Mathias, A. \& Corthésy, B. Recognition of Gram-positive intestinal bacteria by hybridoma- and colostrum-derived secretory immunoglobulin $A$ is mediated by carbohydrates. J. Biol. Chem. 286, 17239-17247 (2011).

33. Nakajima, A. et al. IgA regulates the composition and metabolic function of gut microbiota by promoting symbiosis between bacteria. J. Exp. Med. 215, 2019-2034 (2018).

34. Al Nabhani, Z. et al. A weaning reaction to microbiota is required for resistance to immunopathologies in the adult. Immunity 50, 1276-1288.e5 (2019).

35. Abdel-Gadir, A. et al. Microbiota therapy acts via a regulatory T cell MyD88/ RORyt pathway to suppress food allergy. Nat. Med. 25, 1164-1174 (2019).

36. Mora, J. R. et al. Generation of gut-homing IgA-secreting B cells by intestinal dendritic cells. Science 314, 1157-1160 (2006).

37. Tan, J. et al. Dietary fiber and bacterial SCFA enhance oral tolerance and protect against food allergy through diverse cellular pathways. Cell Rep. 15, 2809-2824 (2016).

38. Kim, M., Qie, Y., Park, J. \& Kim, C. H. Gut microbial metabolites fuel host antibody responses. Cell Host Microbe 20, 202-214 (2016).

39. $\mathrm{Wu}, \mathrm{W}$. et al. Microbiota metabolite short-chain fatty acid acetate promotes intestinal IgA response to microbiota which is mediated by GPR43. Mucosal Immunol. 10, 946-956 (2017).

40. Sanchez, H. N. et al. B cell-intrinsic epigenetic modulation of antibody responses by dietary fiber-derived short-chain fatty acids. Nat. Commun. 11, 60 (2020).

41. Stefka, A. T. et al. Commensal bacteria protect against food allergen sensitization. Proc. Natl Acad. Sci. USA 111, 13145-13150 (2014).

42. Wang, Y. et al. An LGG-derived protein promotes $\lg A$ production through upregulation of APRIL expression in intestinal epithelial cells. Mucosal Immunol. 10, 373-384 (2017).

43. Shen, X. et al. Supplementation of p40, a Lactobacillus rhamnosus GG-derived protein, in early life promotes epidermal growth factor receptor-dependent intestinal development and long-term health outcomes. Mucosal Immunol. 11, 1316-1328 (2018).

44. Fehres, C. M. et al. APRIL induces a novel subset of $\lg A+$ regulatory $B$ cells that suppress inflammation via expression of IL-10 and PD-L1. Front. Immunol. 10, 1368 (2019).

45. Aitoro, R. et al. Extensively hydrolyzed casein formula alone or with L. rhamnosus GG reduces $\beta$-lactoglobulin sensitization in mice. Pediatr. Allergy Immunol. 28, 230-237 (2017).

46. Nocerino, R. et al. Dietary treatment with extensively hydrolyzed casein formula containing the probiotic Lactobacillus rhamnosus GG prevents the occurrence of functional gastrointestinal disorders in children with cow's milk allergy. J. Pediatr. 213, 137-142.e2 (2019).
47. Cho, H. et al. Defective IgA response to atypical intestinal commensals in IL-21 receptor deficiency reshapes immune cell homeostasis and mucosal immunity. Mucosal Immunol. 12, 85-96 (2019).

48. Cong, Y. \& Li, Y. Different flavors of IL-21 in regulation of intestinal IgA to commensals. Mucosal Immunol. 12, 36-38 (2019).

49. Hara, S. et al. Dietary antigens induce germinal center responses in peyer's patches and antigen-specific IgA production. Front. Immunol. 10, 2432 (2019).

50. Palm, N. W. et al. Immunoglobulin A coating identifies colitogenic bacteria in inflammatory bowel disease. Cell 158, 1000-1010 (2014).

51. Viladomiu, M. et al. IgA-coated $E$. coli enriched in Crohn's disease spondyloarthritis promote TH17-dependent inflammation. Sci. Transl. Med. 9, eaaf9655 (2017).

52. Zhang, B. et al. Divergent $T$ follicular helper cell requirement for $\lg A$ and $\lg E$ production to peanut during allergic sensitization. Sci. Immunol. 5, eaay2754 (2020).

53. Aghamohammadi, A et al. IgA deficiency: correlation between clinical and immunological phenotypes. J. Clin. Immunol. 29, 130-136 (2009).

54. Lougaris, V. et al. Clinical and laboratory features of 184 Italian pediatric patients affected with selective IgA deficiency (SlgAD): a Longitudinal Single-Center Study. J. Clin. Immunol. 39, 470-475 (2019).

55. Orivuori, L. et al. Immunoglobulin $A$ and immunoglobulin $\mathrm{G}$ antibodies against $\beta$-lactoglobulin and gliadin at age 1 associate with immunoglobulin E sensitization at age 6. Pediatr. Allergy Immunol. 25, 329-337 (2014).

56. Konstantinou, G. N. et al. Egg-white-specific $\lg A$ and $\lg A 2$ antibodies in eggallergic children: is there a role in tolerance induction? Pediatr. Allergy Immunol. 25, 64-70 (2014).

57. Kim, K. S. et al. Dietary antigens limit mucosal immunity by inducing regulatory T cells in the small intestine. Science 351, 858-863 (2016).

58. Reber, L. L., Hernandez, J. D. \& Galli, S. J. The pathophysiology of anaphylaxis. J. Allergy Clin. Immunol. 140, 335-348 (2017).

59. Harris, N. L. \& Loke, P. Recent advances in type-2-cell-mediated. Immunity 47, 1024-1036 (2017)

60. Brosschot, T. P. \& Reynolds, L. A. The impact of a helminth-modified microbiome on host immunity. Mucosal Immunol. 11, 1039-1046 (2018).

61. Gieseck, R. L., Wilson, M. S. \& Wynn, T. A. Type 2 immunity in tissue repair and fibrosis. Nat. Rev. Immunol. 18, 62-76 (2018).

62. Maizels, R. M. \& McSorley, H. J. Regulation of the host immune system by helminth parasites. J. Allergy Clin. Immunol. 138, 666-675 (2016).

63. Kelly, B. T. \& Grayson, M. H. Immunoglobulin E, what is it good for? Ann. Allergy Asthma Immunol. 116, 183-187 (2016).

64. Anvari, S., Miller, J., Yeh, C.-Y. \& Davis, C. M. IgE-mediated food allergy. Clin. Rev. Allergy Immunol. 57, 244-260 (2019).

65. Sato, S., Yanagida, N. \& Ebisawa, M. How to diagnose food allergy. Curr. Opin. Allergy Clin. Immunol. 18, 214-221 (2018).

66. Shade, K.-T. C. et al. Sialylation of immunoglobulin E is a determinant of allergic pathogenicity. Nature 582, 265-270 (2020).

67. Beyer, K. et al. Predictive values of component-specific lgE for the outcome of peanut and hazelnut food challenges in children. Allergy 70, 90-98 (2015).

68. Ebisawa, M., Shibata, R., Sato, S., Borres, M. P. \& Ito, K. Clinical utility of IgE antibodies to $\omega-5$ gliadin in the diagnosis of wheat allergy: a pediatric multicenter challenge study. Int. Arch. Allergy Immunol. 158, 71-76 (2012).

69. Lange, $\mathrm{L}$. et al. Ana o 3 -specific lgE is a good predictor for clinically relevant cashew allergy in children. Allergy 72, 598-603 (2017).

70. Caubet, J. C. et al. Natural tolerance development in cow's milk allergic children: IgE and lgG4 epitope binding. Allergy 72, 1677-1685 (2017).

71. Suárez-Fariñas, M. et al. Predicting development of sustained unresponsiveness to milk oral immunotherapy using epitope-specific antibody binding profiles. J. Allergy Clin. Immunol. 143, 1038-1046 (2019).

72. Chipps, B. E. et al. Omalizumab in children with uncontrolled allergic asthma: Review of clinical trial and real-world experience. J. Allergy Clin. Immunol. 139 1431-1444 (2017).

73. Leung, D. Y. M. et al. Effect of anti-lgE therapy in patients with peanut allergy. $N$. Engl. J. Med. 348, 986-993 (2003).

74. Andorf, S. et al. Anti-lgE treatment with oral immunotherapy in multifood allergic participants: a double-blind, randomised, controlled trialt. Lancet Gastroenterol. Hepatol. 3, 85-94 (2018).

75. Moñino-Romero, $\mathrm{S}$. et al. The soluble isoform of human $\mathrm{Fc \varepsilon RI}$ is an endogenous inhibitor of IgE-mediated mast cell responses. Allergy 74, 236-245 (2019).

76. Levin, M., Levander, F., Palmason, R., Greiff, L. \& Ohlin, M. Antibody-encoding repertoires of bone marrow and peripheral blood-a focus on IgE. J. Allergy Clin. Immunol. 139, 1026-1030 (2017).

77. Heeringa, J. J. et al. IgE-expressing memory B cells and plasmablasts are increased in blood of children with asthma, food allergy, and atopic dermatitis. Allergy 73, 1331-1336 (2018). 
78. Hoh, R. A. et al. Origins and clonal convergence of gastrointestinal lgE $+B$ cells in human peanut allergy. Sci. Immunol. 5, eaay4209 (2020).

79. Wesemann, D. R. \& Nagler, C. R. Origins of peanut allergy-causing antibodies. Science 367, 1072-1073 (2020).

80. Looney, T. J. et al. Human B-cell isotype switching origins of IgE. J. Allergy Clin. Immunol. 137, 579-586.e7 (2016).

81. Wesemann, D. R. et al. Immature B cells preferentially switch to IgE with increased direct $S \mu$ to $S \varepsilon$ recombination. J. Exp. Med. 208, 2733-2746 (2011).

82. He, J.-S. et al. IgG1 memory B cells keep the memory of IgE responses. Nat. Commun. 8, 641 (2017).

83. Xiong, H., Dolpady, J., Wabl, M., Curotto de Lafaille, M. A. \& Lafaille, J. J. Sequential class switching is required for the generation of high affinity lgE antibodies. J. Exp. Med. 209, 353-364 (2012).

84. Erazo, A. et al. Unique maturation program of the IgE response in vivo. Immunity 26, 191-203 (2007)

85. Hong, S.-W. et al. Food antigens drive spontaneous IgE elevation in the absence of commensal microbiota. Sci. Adv. 5, eaaw1507 (2019).

86. Zimmermann, P., Messina, N., Mohn, W. W., Finlay, B. B. \& Curtis, N. Association between the intestinal microbiota and allergic sensitization, eczema, and asthma: a systematic review. J. Allergy Clin. Immunol. 143, 467-485 (2019).

87. Barcik, W., Boutin, R. C. T., Sokolowska, M. \& Finlay, B. B. The role of lung and gut microbiota in the pathology of asthma. Immunity 52, 241-255 (2020).

88. Bachert, C. et al. Specific IgE against Staphylococcus aureus enterotoxins: an independent risk factor for asthma. J. Allergy Clin. Immunol. 130, 376-381.e8 (2012).

89. Hahn, D. L. et al. Chlamydia pneumoniae-specific lgE is prevalent in asthma and is associated with disease severity. PLOS ONE 7, e35945 (2012).

90. Sørensen, M. et al. Staphylococcus aureus enterotoxin sensitization is associated with allergic poly-sensitization and allergic multimorbidity in adolescents. Allergy 72, 1548-1555 (2017).

91. Dzoro, S. et al. House dust mites as potential carriers for IgE sensitization to bacterial antigens. Allergy 73, 115-124 (2018).

92. Wyss, M. et al. Using precisely defined in vivo microbiotas to understand microbial regulation of IgE. Front. Immunol. 10, 3107 (2020).

93. Feehley, T. et al. Healthy infants harbor intestinal bacteria that protect against food allergy. Nat. Med. 25, 448-453 (2019).

94. Pone, E. J. et al. BCR-signalling synergizes with TLR-signalling for induction of AID and immunoglobulin class-switching through the non-canonical NF-KB pathway. Nat. Commun. 3, 767 (2012).

95. Pone, E. J. et al. B cell TLR1/2, TLR4, TLR7 and TLR9 interact in induction of class switch DNA recombination: modulation by $B C R$ and $C D 40$, and relevance to Tindependent antibody responses. Autoimmunity 48, 1-12 (2015).

96. Bashir, M. E. H., Louie, S., Shi, H. N. \& Nagler-Anderson, C. Toll-like receptor 4 signaling by intestinal microbes influences susceptibility to food allergy. $J$. Immunol. 172, 6978-6987 (2004).

97. Ganley-Leal, L. M., Liang, Y., Jagannathan-Bogdan, M., Farraye, F. A. \& Nikolajczyk, B. S. Differential regulation of TLR4 expression in human B cells and monocytes. Mol. Immunol. 48, 82-88 (2010).

98. Crespo-Lessmann, A. et al. Expression of toll-like receptors 2 and 4 in subjects with asthma by total serum IgE level. Respir. Res. 17, 41 (2016).

99. Tropini, C. et al. Transient osmotic perturbation causes long-term alteration to the gut microbiota. Cell 173, 1742-1754.e17 (2018).

100. Noval Rivas, M. et al. A microbiota signature associated with experimental food allergy promotes allergic sensitization and anaphylaxis. J. Allergy Clin. Immunol. 131, 201-212 (2013)

101. Price, A. E. et al. A map of toll-like receptor expression in the intestinal epithelium reveals distinct spatial, cell type-specific, and temporal patterns. Immunity 49, 560-575.e6 (2018),

102. Whitehead, G. S. et al. TLR5 activation exacerbates airway inflammation in asthma. Lung 198, 289-298 (2020).

103. Li, L.-J. et al. Flagellin modulates IgE expression in B cells to initiate food allergy in mice. Am. J. Transl. Res. 8, 2748-2757 (2016).

104. Shim, J.-U. et al. Flagellin suppresses experimental asthma by generating regulatory dendritic cells and T cells. J. Allergy Clin. Immunol. 137, 426-435 (2016).

105. Van Maele, L. et al. TLR5 signaling stimulates the innate production of IL-17 and IL-22 by CD3(neg)CD127+ immune cells in spleen and mucosa. J. Immunol. 185, 1177-1185 (2010).

106. Kinnebrew, M. A. et al. Interleukin 23 production by intestinal CD103(+)CD11b $(+)$ dendritic cells in response to bacterial flagellin enhances mucosal innate immune defense. Immunity 36, 276-287 (2012).

107. Flores-Langarica, A. et al. Intestinal CD103+CD11b+ CDC2 conventional dendritic cells are required for primary $C D 4+T$ and $B$ cell responses to soluble flagellin. Front. Immunol. 9, 2409 (2018).
108. Schülke, S. et al. A fusion protein of flagellin and ovalbumin suppresses the $\mathrm{TH} 2$ response and prevents murine intestinal allergy. J. Allergy Clin. Immunol. 128, 1340-1348.e12 (2011).

109. Schülke, S. Induction of Interleukin-10 producing dendritic cells as a tool to suppress allergen-specific T helper 2 responses. Front. Immunol. 9, 455 (2018).

110. Valenta, R., Hochwallner, H., Linhart, B. \& Pahr, S. Food allergies: the basics. Gastroenterology 148, 1120-1131.e4 (2015).

111. Pali-Schöll, I., Untersmayr, E., Klems, M. \& Jensen-Jarolim, E. The effect of digestion and digestibility on allergenicity of food. Nutrients 10, 1129 (2018).

112. Lee, Y.-S. et al. Microbiota-derived lactate accelerates intestinal stem-cellmediated epithelial development. Cell Host Microbe 24, 833-846.e6 (2018).

113. Nadjsombati, M. S. et al. Detection of succinate by intestinal tuft cells triggers a type 2 innate immune circuit. Immunity 49, 33-41.e7 (2018).

114. Khodoun, M. V., Strait, R., Armstrong, L., Yanase, N. \& Finkelman, F. D. Identification of markers that distinguish IgE- from IgG-mediated anaphylaxis. Proc. Natl Acad. Sci. USA 108, 12413-12418 (2011).

115. Finkelman, F. D., Khodoun, M. V. \& Strait, R. Human IgE-independent systemic anaphylaxis. J. Allergy Clin. Immunol. 137, 1674-1680 (2016).

116. Jönsson, F. et al. An lgG-induced neutrophil activation pathway contributes to human drug-induced anaphylaxis. Sci. Transl. Med. 11, eaat1479 (2019).

117. Nunes, M. P. O., Tilburg, M. F., van, Tramontina, Florean, E. O. P. \& Guedes, M. I. F. Detection of serum and salivary $\lg E$ and $\lg \mathrm{G} 1$ immunoglobulins specific for diagnosis of food allergy. PLoS ONE 14, e0214745 (2019).

118. Orengo, J. M. et al. Treating cat allergy with monoclonal IgG antibodies that bind allergen and prevent IgE engagement. Nat. Commun. 9, 1421 (2018).

119. van de Veen, W et al. IgG4 production is confined to human IL-10-producing regulatory $B$ cells that suppress antigen-specific immune responses. J. Allergy Clin. Immunol. 131, 1204-1212 (2013).

120. Aalberse, R. C., van der Gaag, R \& van Leeuwen, J. van Serologic aspects of IgG4 antibodies. I. Prolonged immunization results in an IgG4-restricted response. J. Immunol. 130, 722-726 (1983).

121. Burton, O. T. et al. Oral immunotherapy induces IgG antibodies that act through FcyRllb to suppress IgE-mediated hypersensitivity. J. Allergy Clin. Immunol. 134, 1310-1317.e6 (2014).

122. Uotila, R., Kukkonen, A. K., Greco, D., Pelkonen, A. S. \& Mäkelä, M. J. Peanut oral immunotherapy increases IgG4 to Ara h 1, 2, and 6 but does not affect lgG4 to other allergens. Pediatr. Allergy Immunol. 30, 248-252 (2019).

123. Bedoret, D. et al. Changes in antigen-specific T-cell number and function during oral desensitization in cow's milk allergy enabled with omalizumab. Mucosal Immunol. 5, 267-276 (2012).

124. Brandström, J. et al. Individually dosed omalizumab facilitates peanut oral immunotherapy in peanut allergic adolescents. Clin. Exp. Allergy J. 49, 1328-1341 (2019).

125. Stranks, A. J. et al. Immunoglobulin E blockade during food allergen ingestion enhances the induction of inhibitory immunoglobulin $\mathrm{G}$ antibodies. Ann. Allergy Asthma Immunol. 122, 213-215 (2019).

126. Lilienthal, G.-M. et al. Potential of murine $\lg G 1$ and human $\lg G 4$ to inhibit the classical complement and Fcy receptor activation pathways. Front. Immunol. 9, 958 (2018).

127. Koneczny, I. Update on IgG4-mediated autoimmune diseases: new insights and new family members. Autoimmun. Rev. 19, 102646 (2020).

128. Bruhns, P. et al. Specificity and affinity of human Fcgamma receptors and their polymorphic variants for human IgG subclasses. Blood 113, 3716-3725 (2009).

129. Smith, K. G. C. \& Clatworthy, M. R. FcgammaRIIB in autoimmunity and infection: evolutionary and therapeutic implications. Nat. Rev. Immunol. 10, 328-343 (2010).

130. Xiao, W. et al. Positive and negative regulation of mast cell activation by Lyn via the FcepsilonRI. J. Immunol. 175, 6885-6892 (2005).

131. Berin, M. C. et al. Egg-specific lgE and basophil activation but not egg-specific Tcell counts correlate with phenotypes of clinical egg allergy. J. Allergy Clin. Immunol. 142, 149-158.e8 (2018).

132. Datema, M. R. et al. Ratios of specific lgG 4 over lgE antibodies do not improve prediction of peanut allergy nor of its severity compared to specific lgE alone. Clin. Exp. Allergy 49, 216-226 (2019).

133. Rosser, E. C. et al. Microbiota-derived metabolites suppress arthritis by amplifying Aryl-hydrocarbon receptor activation in regulatory B cells. Cell Metab. 31, 837-851.e10 (2020).

134. Gao, X. et al. Critical roles of regulatory B and T cells in helminth parasiteinduced protection against allergic airway inflammation. Clin. Exp. Immunol. 198, 390-402 (2019).

135. Piper, C. J. M. et al. Aryl hydrocarbon receptor contributes to the transcriptional program of IL-10-producing regulatory B cells. Cell Rep. 29, 1878-1892.e7 (2019). 
136. Matsushita, T. et al. A novel splenic B1 regulatory cell subset suppresses allergic disease through phosphatidylinositol 3-kinase-Akt pathway activation. J. Allergy Clin. Immunol. 138, 1170-1182.e9 (2016).

137. Rensburg, I. C., van, Kleynhans, L., Keyser, A., Walzl, G. \& Loxton, A. G. B-cells with a FasL expressing regulatory phenotype are induced following successful antituberculosis treatment: TB treatment induces FasL regulatory B cells. Immun. Inflamm. Dis. 5, 57-67 (2017).

138. Wang, $X$. et al. PD-1-expressing $B$ cells suppress $C D 4+$ and $C D 8+T$ cells via PD1/PD-L1-dependent pathway. Mol. Immunol. 109, 20-26 (2019).

139. Yang, G. et al. Thrombospondin-1 (TSP1)-producing B cells restore antigen (Ag)specific immune tolerance in an allergic environment. J. Biol. Chem. 290 12858-12867 (2015)
140. Dawicki, W., Li, C., Town, J., Zhang, X. \& Gordon, J. R. Therapeutic reversal of food allergen sensitivity by mature retinoic acid-differentiated dendritic cell induction of $\mathrm{LAG3}^{+} \mathrm{CD} 4 \mathrm{~b}^{-} \mathrm{Foxp}^{-}$regulatory T cells. J. Allergy Clin. Immunol. 139, 1608-1620.e3 (2017).

141. Mishima, Y. et al. Microbiota maintain colonic homeostasis by activating TLR2/ MyD88/PI3K signaling in IL-10-producing regulatory B cells. J. Clin. Investig. 129, 3702-3716 (2019)

142. Maerz, J. K. et al. Bacterial immunogenicity is critical for the induction of regulatory B cells in suppressing inflammatory immune responses. Front. Immunol. 10, 3093 (2019).

143. Vatanen, T. et al. Variation in microbiome LPS immunogenicity contributes to autoimmunity in humans. Cell 165, 842-853 (2016). 